\title{
Efecto del peróxido de hidrógeno al $25 \%$ sobre la microdureza del esmalte dental
}

\author{
Effectofhydrogen peroxide $25 \%$ on microhardness of dentalenamel
}

\begin{abstract}
Resumen
Se realizó un estudio experimental con la finalidad de evaluar el efecto del peróxido de hidrógeno al $25 \%$ sobre la microdureza superficial del esmalte dental. Se utilizaron 30 premolares extraídos con fines ortodónticos. Estos fueron seccionados y colocados en un molde circunferencial de $1 \mathrm{~cm}$ de diámetro por $1 \mathrm{~cm}$ de espesor rellenándolo con acrílico autopolimerizable de color rojo (15) y azul (15), almacenándolos en frascos estériles debidamente rotulados con solución fisiológica isotónica. Se realizó una medida inicial de la dureza del esmalte empleando el Microdurómetro BUEHLER MICROMET 2101, en el Laboratorio de Sputtering y Alto Vacío de la Facultad de Ciencias de la Universidad Nacional de Ingeniería, aplicando una carga de 100gr durante 30 segundos. Se procedió a medir la indentación para hallar la medida de la microdureza en $\mathrm{Kg} / \mathrm{mm} 2$. Al grupo 1 se le realizó el clareamiento con Peroxgel MCC y al grupo 2 con Zoom2, al día siguiente se volvió a medir la microdureza superficial del esmalte dental. Los datos obtenidos de la microdureza superficial del esmalte para el grupo sometido al agente clareador Peroxgel MCC y Zoom2 presentaron una disminución estadísticamente significativa (234.83 $\mathrm{Kgf} / \mathrm{mm} 2)$ y $(196.11 \mathrm{Kgf} / \mathrm{mm} 2)$ respectivamente, no encontrándose diferencia significativa $(\mathrm{p}=0.136)$ al comparar esta disminución entre los agentes clareadores utilizados en este estudio. Se concluye que el uso de agentes clareadores a base de peróxido de hidrógeno al 25\% disminuye significativamente la microdureza del esmalte dental.
\end{abstract}

Palabra clave: descalcificación, blanqueadores, calcificación.

\begin{abstract}
The aim was determinate the effect of two bleaching agents based on hydrogen peroxide $25 \%$ on dental enamel microhardness. An experimental study was made to evaluate the effect of hydrogen peroxide $25 \%$ on the enamel surface microhardness, it was used 30 extracted premolars with orthodontic purposes, which were sectioned and placed in a mold of $1 \mathrm{~cm}$ circumferential in diameter and $1 \mathrm{~cm}$ thick, filling with acrylic self-curing color red (15) and blue (15) storing in sterile flasks containing physiological serum. An initial measurement was using the BUEHLER MICROMET 2101 microhardness in the Sputtering Laboratory, Faculty of Science, National University of Engineering, applied a load of $100 \mathrm{~g}$ for 30 seconds, then proceeded to measure the indentation to find the measure of microhardness in $\mathrm{kg} / \mathrm{mm} 2$. Then group 1 was done bleaching with Peroxgel MCC and group 2 with Zoom2, the next day again measured enamel microhardness. The results of the enamel surface microhardness of the whitening agent: Peroxgel MCC and Zoom2, showed a statistically significant decrease (234.83 Kgf/ $\mathrm{mm} 2)$ and $(196.11 \mathrm{Kgf} / \mathrm{mm} 2)$ respectively, found no significant difference $(\mathrm{p}=0.136)$ when comparing the decline between bleaching agents used in this study. It is concluded that the use of bleaching agents based on hydrogen peroxide $25 \%$ decreased significantly the microhardness of the enamel surface.
\end{abstract}

Keyword: bleach, calcification, bleaching agents .

\section{Introducción}

La sonrisa es considerada un accesorio fundamental que compone la apariencia y la presentación del individuo en la sociedad. El nuevo patrón estético es presentado por dientes de tonalidades muy claras, bien conformados y correctamente alineados, siendo así que los dientes oscurecidos interfieren en la apariencia de la sonrisa. ${ }^{1}$

El clareamiento dental es una práctica en auge dentro de la odontología clínica, ya que sus propósitos estéticos tienen como objetivo satisfacer las necesidades de los pacientes, quienes tratan de mantenerse saludables y lucir más jóvenes. ${ }^{2}$

Este logra una notable disminución de las pigmentaciones de la estructura superficial del esmalte dental por medio de la descomposición del peróxido en radicales libres inestables, los cuales pueden romper las moléculas mediante reacciones de óxido-reducción que obran sobre los pigmentos depositados en los ameloblastos. ${ }^{2}$

Se ha encontrado en estudios con microscopía electrónica de barrido que el esmalte tratado con peróxido de hidrógeno, da como resultado una ligera
Artículo Original

\section{Vania Sánchez Gamarra ${ }^{1}$, Germán Chávez Zelada²}

1 Cirujano Dentista. Facultad de Odontología Universidad de San Martín de Porres, LimaPerú

2 Doctor en Odontología. Docente de la Facultad de Odontología. Universidad de San Martín de Porres, Lima-Perú.

\section{Correspondencia}

Vania Sánchez Gamarra

Jr. Tomas Ramsey 915 Dpto. 503 Magdalena del mar. Lima-Perú.

Correo electrónico: vaniasg04@gmail.com

Fecha de recepción: 08 de abril

Fecha de aceptación: 14 de junio corrosión de la superficie, produciendo superficies defectuosas similares a la erosión. ${ }^{3-5}$

Los cambios en los estilos de vida con el cada vez más fuerte patrón estético como sinónimo de salud, apoyados por el constante bombardeo de los medios de comunicación visuales, ha traído como consecuencia, el uso indiscriminado de agentes de clareamiento bajo ningún control por parte del odontólogo. ${ }^{6}$

Como respuesta a esta demanda y además por lo antes citado de que la literatura advierte que estos efectos aumentan cuanto mayor es la concentración 
del agente clareador ${ }^{5-7}$, así como cuanto más frecuente y prolongado sea el tratamiento. ${ }^{7-9}$ Se consideró importante llevar a cabo esta investigación evaluando dos agentes clareadores que contengan peróxido de hidrógeno al $25 \%$ y así poder evaluar su efecto sobre la microdureza superficial del esmalte dental.

\section{Material y métodos}

Se realizó un estudio experimental y comparativo para evaluar el efecto del peróxido de hidrógeno al 25\% sobre la microdureza superficial del esmalte dental.

Se recolectó piezas dentarias humanas, las que fueron seleccionadas según los criterios de inclusión (30 premolares extraídas por motivos ortodónticos). Posteriormente fueron lavadas con agua y jabón líquido y se colocaron en una solución fisiológica isotónica a fin de mantenerlos hidratados.

Se dividieron en dos grupos de 15 piezas y luego se seccionó la corona con un disco de metal colocado en un micromotor de baja velocidad.

Se confeccionaron bases de acrílico de curado rápido usando un molde circunferencial de $1 \mathrm{~cm}$ de diámetro por $1 \mathrm{~cm}$ de espesor, se introdujo la corona de la pieza dentaria en el área superficial a evaluar en la parte superior, se verificó el paralelismo empleando una platina de vidrio y se empleó acrílico transparente de color rojo para el grupo A y de color azul para el grupo B, para diferenciar los especímenes, además se escribió el número de cada uno en la parte inferior para poder identificarlos.

Los especímenes fueron almacenados en frascos estériles debidamente rotulados conteniendo la solución fisiológica isotónica. Estas piezas no presentaron grietas ni líneas de fractura al ser observadas bajo un microscopio de 400 aumentos incorporado al microdurómetro. (Fig. 1)

Se realizó una medida inicial empleando el microdurómetro BUEHLER MICROMET 2101 en el Laboratorio de Sputtering y Alto Vacío de la Facultad de Ciencias de la Universidad Nacional de Ingeniería. El microdurómetro fue programado para aplicar una carga de $100 \mathrm{~g}$ durante 30 segundos. Con el microscopio incorporado se buscó un área regular de esmalte donde se realizará la indentación midiéndose la diagonal para hallar la medida de la microdureza en $\mathrm{kg} / \mathrm{mm} 2$, empleando la fórmula:

$$
\mathrm{HV}=1840 \frac{\mathrm{L}}{\mathrm{D}^{2}} \mathrm{~kg} / \mathrm{mm}^{2}
$$

Los 15 especímenes de cada grupo fueron colocados en recipientes rotulados, después de realizar la profilaxis mediante el uso de piedra pómez y agua. Se expusieron a la acción de los agentes clareadores: Peroxgel MCC y Zoom2 respectivamente, según las indicaciones de cada fabricante.

En el grupo A se usó el agente clareador Peroxgel MCC, el cual tiene por indicación aplicarlo en tres sesiones continuas de veinte minutos cada una, expuestos a luz Led, los cuales fueron lavados a presión con agua destilada después de cada aplicación del agente clareador. Posteriormente se aplicó el neutralizador (fosfato de bicarbonato de sodio) por 15 minutos. (Fig. 2)

En el grupo B se usó el agente clareador Zoom2, el cual tiene por indicación aplicarlo en tres sesiones continuas de quince minutos cada una, expuestos a la luz Zoom, los cuales fueron lavados a presión con agua destilada después de cada aplicación de agente clareador. Posteriormente se aplicó fosfato de calcio amorfo (ACP), por 30 minutos. (Fig. 3). Ambos grupos se mantuvieron a temperatura ambiente y luego cado grupo se almacenó en un frasco con solución fisiológica isotónica respectivamente.

Después de haber terminado el clareamiento dental de ambos grupos se volvió a medir la microdureza superficial de los 30 especímenes siguiendo el mismo método aplicado para la medición inicial. (Fig. 4)

Se utilizó el paquete estadístico SPSS Versión 20.0, se realizaron los cálculos de desviación estándar.

Tabla 1. Comparación de la microdureza antes y después del uso de los agentes clareadores PEROXGEL MCC y ZOOM2.

Agentes Clareadores Media $\left(\mathrm{Kgf} / \mathrm{mm}^{2}\right)$ Desviación estándar

\begin{tabular}{cccc}
\hline & Antes & Después & Disminución \\
Peroxgel MCC & $405.08^{*}( \pm)$ & $170.25^{*}( \pm)$ & $234.83^{* * *}( \pm)$ \\
ZOOM2 & $369.5^{* *}( \pm)$ & $173.39^{* *}( \pm)$ & $196.11^{* * *}( \pm)$ \\
\hline T de Student & $\mathrm{p}^{*} 0.000$ & $\mathrm{p}^{* *} 0.000$ & $\mathrm{p}^{* * *} 0.136$
\end{tabular}

La comparación entre la microdureza inicial y final de cada espécimen en cada grupo se realizó mediante la prueba "t de Student" para muestras pareadas y para la comparación de la microdureza final entre los grupos se realizó la prueba "t de Student" para muestras normales.

El tratamiento de los datos se efectuó a nivel de confianza de $95 \%$ y con un margen de error del 5\%.

\section{Resultados}

Después de evaluar los datos obtenidos de la microdureza superficial del esmalte para el grupo sometido al agente clareador Peroxgel MCC del Laboratorio Euroquim, se observa que la media antes del agente se encontraba en 405.08 $\mathrm{Kgf} / \mathrm{mm} 2( \pm)$, disminuyendo después del uso a $170.25 \mathrm{Kgf} / \mathrm{mm} 2( \pm)$. Disminución estadísticamente significativa $\mathrm{p}=0.000$. (Tabla 1)

Después de evaluar los datos obtenidos de la microdureza superficial del esmalte para el grupo sometido al agente clareador Zoom2 de la casa Discus Dental se observa que la media antes del agente se encontraba en $369.5 \mathrm{Kgf} / \mathrm{mm} 2$, disminuyendo después del uso a 173.39 $\mathrm{Kgf} / \mathrm{mm} 2$. Disminución estadísticamente significativa $\mathrm{p}=0.000$. (Tabla 1 )

Después de comparar los datos obtenidos de la microdureza superficial del esmalte para el grupo sometido al agente clareador Peroxgel MCC del Laboratorio Euroquim y Zoom2 de la casa Discus Dental se observa que si bien la disminución de la microdureza del esmalte dental fue mayor para el agente Peroxgel MCC $(234.83 \mathrm{Kgf} / \mathrm{mm} 2 \pm)$ respecto al Zoom2 $(196.11 \mathrm{Kgf} / \mathrm{mm} 2 \pm)$ ésta no es estadísticamente significativa $(\mathrm{p}=$ 0.136). (Tabla 1) 


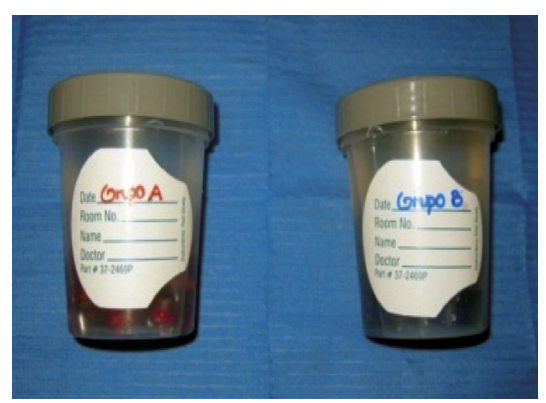

Fig. 1. Frascos rotulados, conteniendo especímenes en solución fisiológica isotónica.

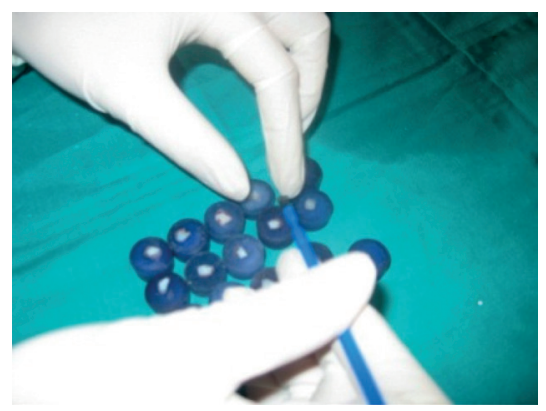

Fig. 3. Aplicación del agente clareador Zoom2.

\section{Discusión}

Oteo et $\mathrm{al}^{10}$ realizaron un estudio en 30 pacientes para valorar la eficacia clínica del clareamiento producido por la reacción foto-fenton, con peróxido de hidrógeno al $25 \%$, activado con luz ultravioleta y compararlo con la eficacia clínica cuando no se aplica luz ultravioleta. Concluyeron que el tratamiento con peróxido de hidrógeno sin activación de luz mostró mínimos cambios en el color al medirlo inmediatamente.10 Sin embargo, al medirlo después de la aplicación de luz se observaron cambios significativos incluso en los dientes a los que se les bloqueó la luz con una barrera opaca. ${ }^{10}$

Este estudio evalúa el sistema Zoom2, uno de los geles de clareamiento utilizados en el presente estudio, el cual emite una luz ultravioleta para activar el gel de peróxido de hidrógeno que es catalizada por una reacción foto-fenton. Este gel contiene gluconato ferroso el cual activa el peróxido, realizándose la reacción de fenton $(\mathrm{Fe} 2+\mathrm{H} 2 \mathrm{O} 2 \mathrm{Fe} 3+$ $\mathrm{OH}-+\mathrm{OH})$. Formando radicales libres oxhidrilos o hidroxilos que son muy reactivos, rompen los enlaces dobles de carbono, convirtiéndose los cromófobos en simples o anulándolos (parte responsable del color de una estructura), dándose de esta forma el clareamiento. El punto controversial en cuanto a que si usar luz ultravioleta para la cataliza-

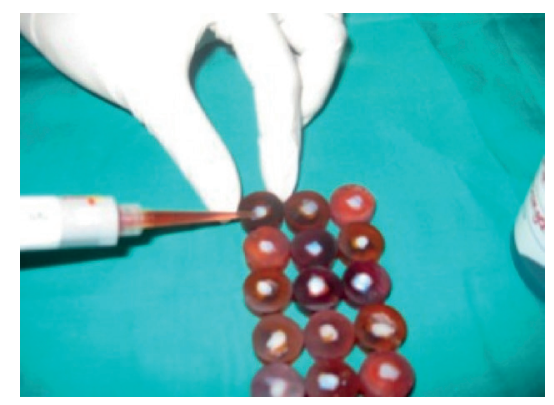

Fig. 2. Aplicación del agente clareador Peroxgel MCC.

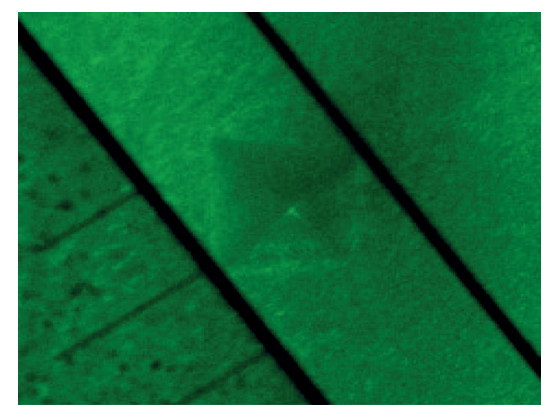

Fig. 4. Identación Final vista desde el lente óptico del Microdurómetro de Vickers, con un aumento de 4.0.

ción de la reacción química del clareamiento es beneficioso o no. En la literatura no existe suficiente información respecto a los efectos que se producen en la estructura de los dientes bajo la luz ultravioleta, porque si bien es cierto el efecto se magnifica por otro lado las consecuencias sobre la sensibilidad dental manifiesta durante y después del tratamiento, la pérdida leve de la estructura superficial del esmalte, erosiones, fracturas, y otras circunstancias que pueden predisponer al paciente, como complicaciones operatorias, que inclusive pueden facilitar la presencia de caries por perdida de tejido dentario, por todas estas razones hacen que el sistema Zoom2 recomienda el uso de fosfato de calcio amorfo (ACP), para tentar revertir el cuadro además del desensibilizador a base de Flúor y Nitrato de potasio. Mientras que el sistema de Peroxgel MCC, intenta hacer lo mismo pero en condiciones diferentes al no recomendar el uso de una fuente de luz, o si se desea usar para potenciar la acción del gel clareador, recomienda el uso de LEDS, ya que por su menor potencia da una menor temperatura. Este sistema recomienda el uso del neutralizador, a base de bicarbonato de sodio más fosfato de calcio, para promover también la re-mineralización.

Polydorou et al. ${ }^{11}$ estudiaron tres concentraciones diferentes de peróxido de hidrógeno: 38\%, 30\% y 25\%. Encon- trando que la microdureza de Knoop evaluada antes y después de 15 minutos de clareamiento, no presentaba diferencia significativa.11 A diferencia en este estudio se pudo apreciar que la muestra que fue clareada con Peroxgel MCC presentó una disminución de 234.83 $\mathrm{Kgf} / \mathrm{mm} 2$ y la muestra clareada con Zoom2 presentó una disminución de $196.11 \mathrm{Kgf} / \mathrm{mm} 2$.

Tapia et al. ${ }^{12}$ Realizaron un estudio con la finalidad de determinar el efecto del peróxido de hidrógeno al 25\% y luz ultravioleta sobre la micromorfología superficial del esmalte. Se evidenció diferencias estadísticamente significativas, a pesar del uso fluoruro de sodio al $1.1 \%$, ya que este no produce efectos substanciales en la remineralización del tejido expuesto.12 Estos resultados concuerdan con los datos hallados en este estudio donde se pudo observar una disminución estadísticamente significativa en la microdureza superficial del esmalte dental.

Cabrera et al. ${ }^{13}$ El objetivo de este estudio fue evaluar los efectos en esmalte dental después del tratamiento con peróxido de hidrógeno al $25 \%$ y peróxido de hidrógeno al 16\% como sistemas de clareamiento y posterior aplicación de flúor.13 Se concluye que es indispensable el uso de medicamentos posttratamiento (ACP, flúor o nitrato de potasio), como en el presente estudio se recomienda el uso de remineralizadores a base de calcio y fosfato, para cubrir los defectos y así incrementar la deposición de minerales en la superficie del esmalte dental, los cuales tienen potencial para lograr el efecto de formar hidroxiapatita, haciendo la dentina menos sensible, más fuerte y con apariencia continua.

Se debe tomar en cuenta que cuando el diente entra en contacto con la saliva. El tampón fosfato, juega un papel fundamental en situaciones cuando el $\mathrm{pH}$ se reduce por debajo del $\mathrm{pH}$ crítico $(5,5)$, considerándose muy cerca del $\mathrm{pH}$ del peróxido de hidrógeno al 25\% entre 4 y 5 , de esta manera los fosfatos liberados tratan de restablecer el equilibrio perdido, lo que dependerá en último término del contenido de iones de fosfato y calcio del medio circundante, ya que al provocar una baja en estas condiciones se produce una disolución química del esmalte (erosión) y los mecanismos tampón también se ponen en marcha para normalizar el $\mathrm{pH}$ lo antes posible.

La película adquirida es una película orgánica, que se forma de manera natural en la superficie dentaria, es de origen 
salival, que se produce por depósitos de glucoproteínas presentes en la saliva a los pocos minutos del cepillado dental. ${ }^{14}$ Tiene una función protectora, ya que evita la descalcificación dentaria y la penetración de ácidos. ${ }^{14}$ Su formación hace que carbonato de calcio vuelva al diente y regula los ácidos procedentes de la alimentación o formados durante el metabolismo microbiano, previniendo de tal modo la desmineralización, así como también provee un medio para el intercambio de iones calcio, fosfatos y fluoruros durante los procesos de remineralización. Por lo tanto este también es un factor importante ya que en el clareamiento dental hay una degradación de las moléculas de carbono, dando como consecuencia una desmineralización. ${ }^{14}$

\section{Conclusiones}

Se concluye que el uso de clareadores dentales a base de peróxido de hidrógeno al 25\% disminuye significativamente la microdureza superficial del esmalte.

El agente clareador Peroxgel MCC disminuye en mayor proporción la microdureza superficial del esmalte en relación al agente clareador Zoom2 pero esta diferencia no es estadísticamente significativa.

El uso fosfato de calcio amorfo en el caso del sistema Zoom2, de alguna manera ayudo a restablecer la microdureza del esmalte dental.

El uso fosfato de bicarbonato de sodio (neutralizador), en el caso del Peroxgel MCC, de alguna manera ayudo a restablecer la microdureza del esmalte dental.

\section{Referencias bibliográficas}

1. Cadenaro M, Ottavia Ch, Mazzoni A, Nucci C, Matis B, Di -Lenarda R, Breschi L. An in vivo study of the effect of a 38 percent hydrogen peroxide in-office whitening agent on enamel. J Am Dent Assoc. 2010; 14(4):449-54.

2. Berga Caballero A, Navarro L, Amengual Lorenzo J. Evaluación in vivo de los efectos del peróxido de carbamida al $10 \%$ y del peróxido de hidrogeno al 3.5\% sobre la superficie del esmalte. Med Oral. 2007; 12(1):6-9.

3. Mancera A, Cornejo M, Méndez $\mathrm{R}$, Escalante $\mathrm{S}$, Tinoco V, Luna C. Efecto del blanqueamiento con peróxido de hidrógeno al 38\% sobre la microestructura del esmalte dental. Rev Oral. 2011; 12(36):68790 .

4. Álvares F, Carpena G, Cardoso L, Araujo É. Effect of hydrogen peroxide based home bleaching agents on enamel hardness. Braz J Oral Sci. 2006; 5(18):1090-93.

5. Franco C, De Oliveira R, Cavalli V, Giannini M. Efeitos de agentes clareadores à base de peróxidos na microdureza, rugosidade e morfología superficial do esmalte. Braz Oral Res. 2004; 18(4):24-36.

6. Delfino C, Chinelatti M, Carrasco-Guerisoli L, Batista A, Froner I, Palma-Dibb R. Effectiveness of home bleaching agents in discolored teeth and influence on enamel microhardness. J Appl Oral Sci. 2009; 17(4): 57-68.

7. Ushigome T, Takemoto S, Hattori M, Yoshinari M, Kawada E, Oda Y. Influence of peroxide treatment on bovine enamel surface - Crosssectional analysis. Dent Mat J. 2009; 28(3):315-23.

8. Ameri H, Ghavamnasiri M, Abed A. Effects of different bleaching time intervals on fracture tough- ness of enamel. J Conserv Dent. 2010; 14(1):73-5.

9. Sasaki R, Arcanjo A, Florio F, Basting R. Micromorphology and microhardness of enamel after treatment with home-use bleaching agents containing 10\% carbamide peroxide and 7.5\% hydrogen peroxide. J Appl Oral Sci. 2009; 17(6):57-67.

10. Oteo J, Alegría G, Calvo M, Oteo C. Estudio "in vivo" del efecto de la activación con y sin luz ultravioleta del peróxido de hidrógeno al $25 \%$ en el blanqueamiento dental. Cien y prác. 2011; 12(3):128-42.

11. Polydorou O, Hellwing E, Hahn P. The efficacy of three different inoffice bleaching systems and their effect on enamel microhardness. Ope Dent. 2008; 33(5):579-86.

12. Tapia F, Tapia J, Zamorano X, Valenzuela V. Efectos del peróxido de hidrógeno al 25\% y luz ultravioleta sobre la micromorfología superficial del esmalte. Rev dent Ch. 2008; 17(4):57-68.

13. Cabrera A, David M, Pacheco L, Suárez A, Garzón H. Efectos del peróxido de hidrógeno activado con luz ultravioleta y el peróxido de carbamida en aclaramiento dental. Rev est. 2008; 16(1):18-24.

14. Battellino L. Película adquirida salival: revisión de la literatura. Act odont ven. 2007; 45(3):34-7.

\section{Agradecimientos}

Al Mg. Esp Rafael Morales Vadillo por el apoyo del trabajo estadístico y al CD Marcos Cueva Cueto.

\section{Conflicto de interés}

Los autores declaran no tener conflicto de interés en la publicación de este artículo. 